\title{
Significant Efficacy and Long Term Safety Difference with TAF-based STR in Naïve Adults
}

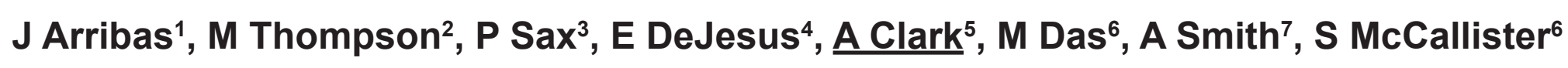

\section{Introduction}

- Two randomized, controlled, double-blinded, multinational Phase 3 trials (Studies 104 [NCT01780506] and 111 [NCT01797445]) disoproxil fumarate (TDF), each in single-tablet regimens coformulated with elvitegravir (E)/cobicistat (C)/emtricitabine $(F)$

- At Weeks 48 (primary endpoint) and 96, E/C/F/TAF had noninferior efficacy (HIV-1 RNA <50 copies/mL) to E/C/F/TDF, and less impact on bone and renal safety ${ }^{2}$

- We now present long-term (144-wk) efficacy, safety, and tolerability of treatment with $E / C / F / T A F$ vs $E / C / F / T D F$ in treatment-naive participants with HIV-1 from these

Prodrug Pharmacology: TAF vs TDF"1,3

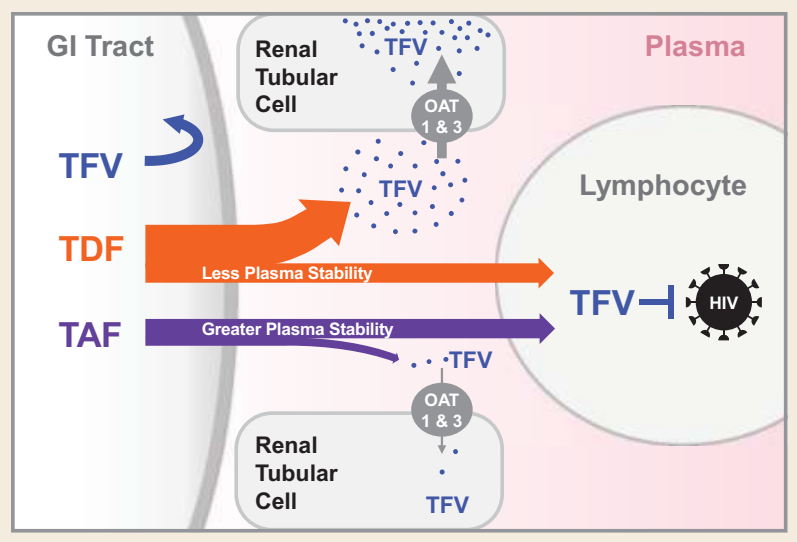

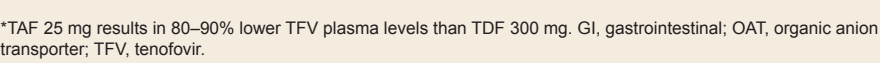

\section{Methods}

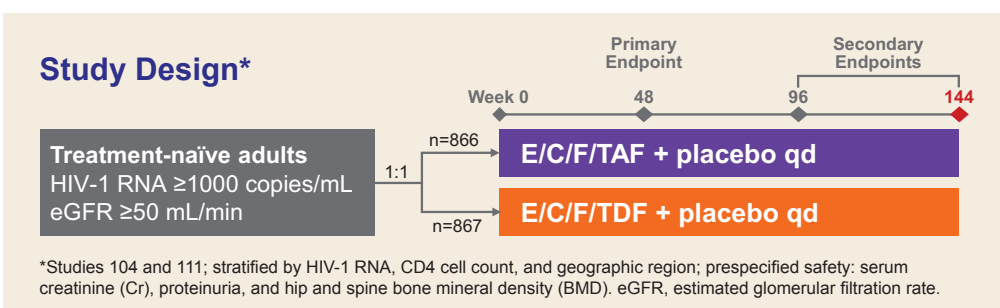

\section{Results}

Baseline Characteristics and Past Medical History

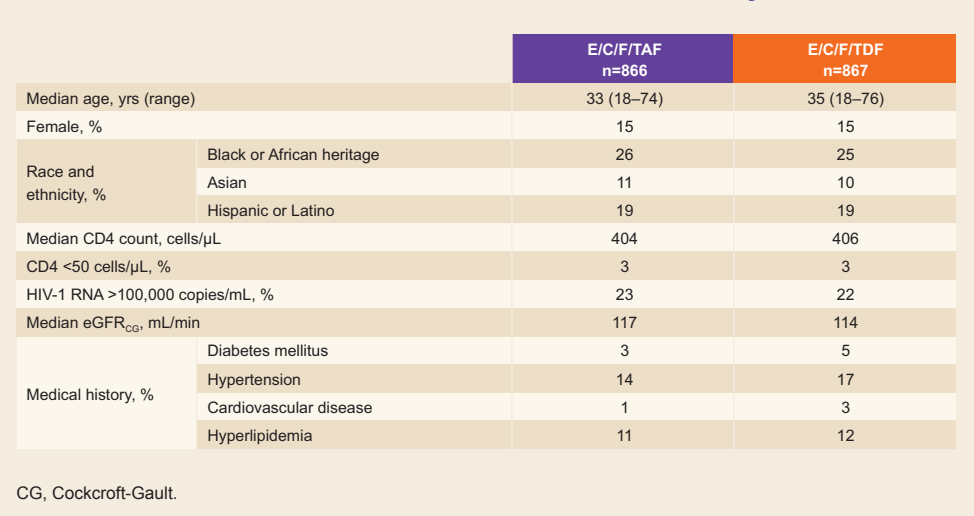

Virologic Outcome at Weeks 48,96 , and $144^{\star 1,2}$

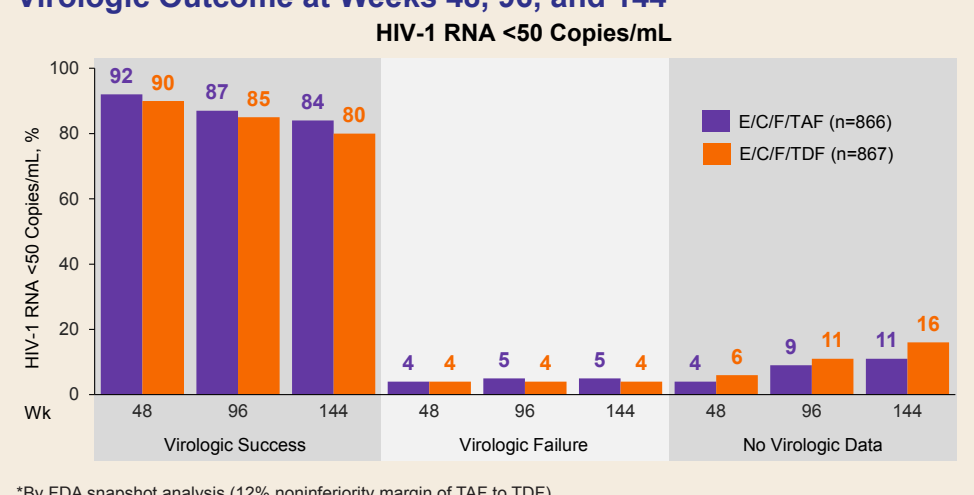

- At Week 144, E/C/F/TAF was superior to E/C/F/TDF in efficacy difference at both $<50$ copies $/ \mathrm{mL}(4.2 \%$ [95\% Cl $0.6 \%, 7.8 \% ; \mathrm{p}=0.02])$ and $<20$ copies $/ \mathrm{mL}$ (5.4\% [95\% Cl 1.5\%, 9.2\%; p=0.01]) Resistance

- By $144 \mathrm{wk}$, virologic failure with resistance occurred in 24 participants $12(1.4 \%)$ on TAF vs $12(1.4 \%)$ on TDF

Genotypic resistance data: NRTI and EVG resistance $(n=8)$ and NRTI (n=7) NRTI resistance only (n=4) an; NRTI and EVG resistance the TDF group

Treatment Difference in Virologic Outcome at Week 144 by Prespecified

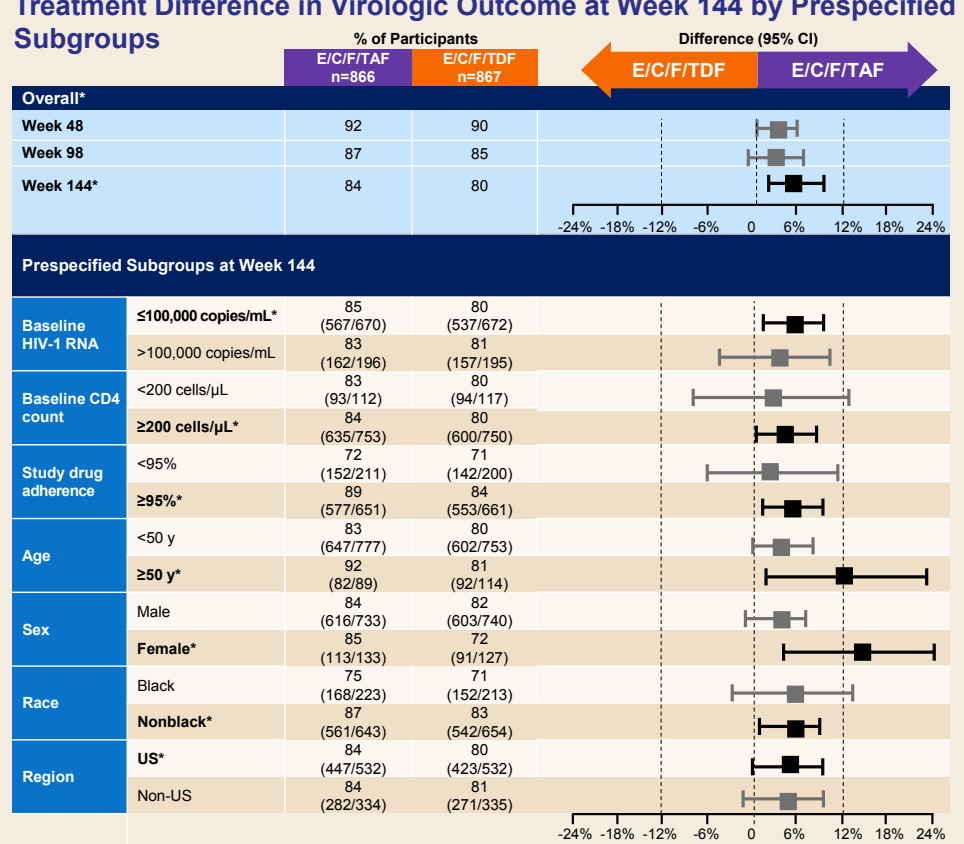

\section{Results (Cont'd)}

\begin{tabular}{|c|c|c|c|}
\hline \multicolumn{4}{|c|}{ Renal Adverse Events Leading to Discontinuation } \\
\hline Renal AE D/C, $\mathrm{n}^{*}$ & $\underset{n=866}{E / C / F / T A F}$ & $\underset{n=867}{E / C / F / T D F}$ & $p$-value \\
\hline Total & 0 & 12 & $<0.001$ \\
\hline Proximal renal tubulopathy ${ }^{\ddagger}$ & 0 & 4 & \\
\hline Increased Cr/decreased eGFR & 0 & 3 & \\
\hline Renal Failure & 0 & 2 & \\
\hline Nephropathy & 0 & 1 & \\
\hline Proteinureia & 0 & 1 & \\
\hline Bladder spasm & 0 & 1 & \\
\hline
\end{tabular}

0 case of proximal renal tubulopathy in E/C/F/TAF arm vs 4 in E/C/F/ TDF arm

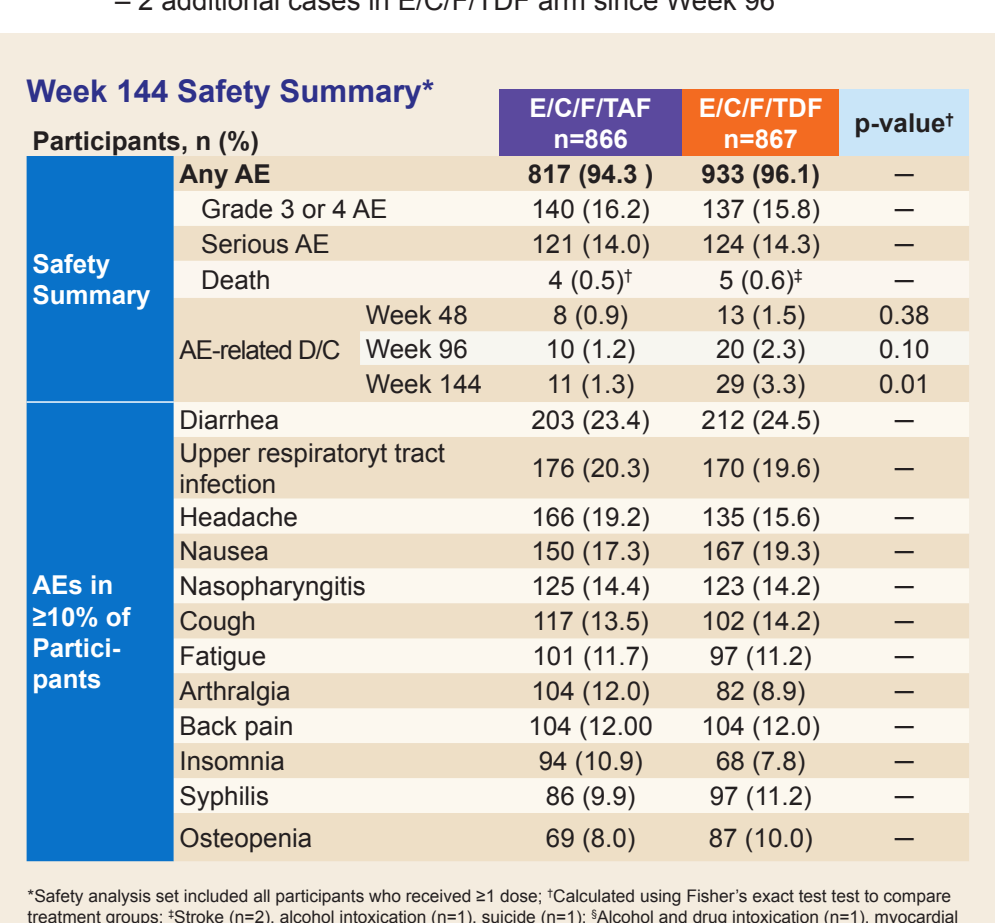

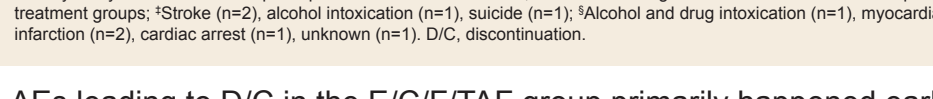
whereas in the E/C/F/TDF group, AEs leading to D/C continued to accumulate, with a significant difference in total number of AEs Week 144

- Most AEs occurred within first 4 wk of treatment initiation

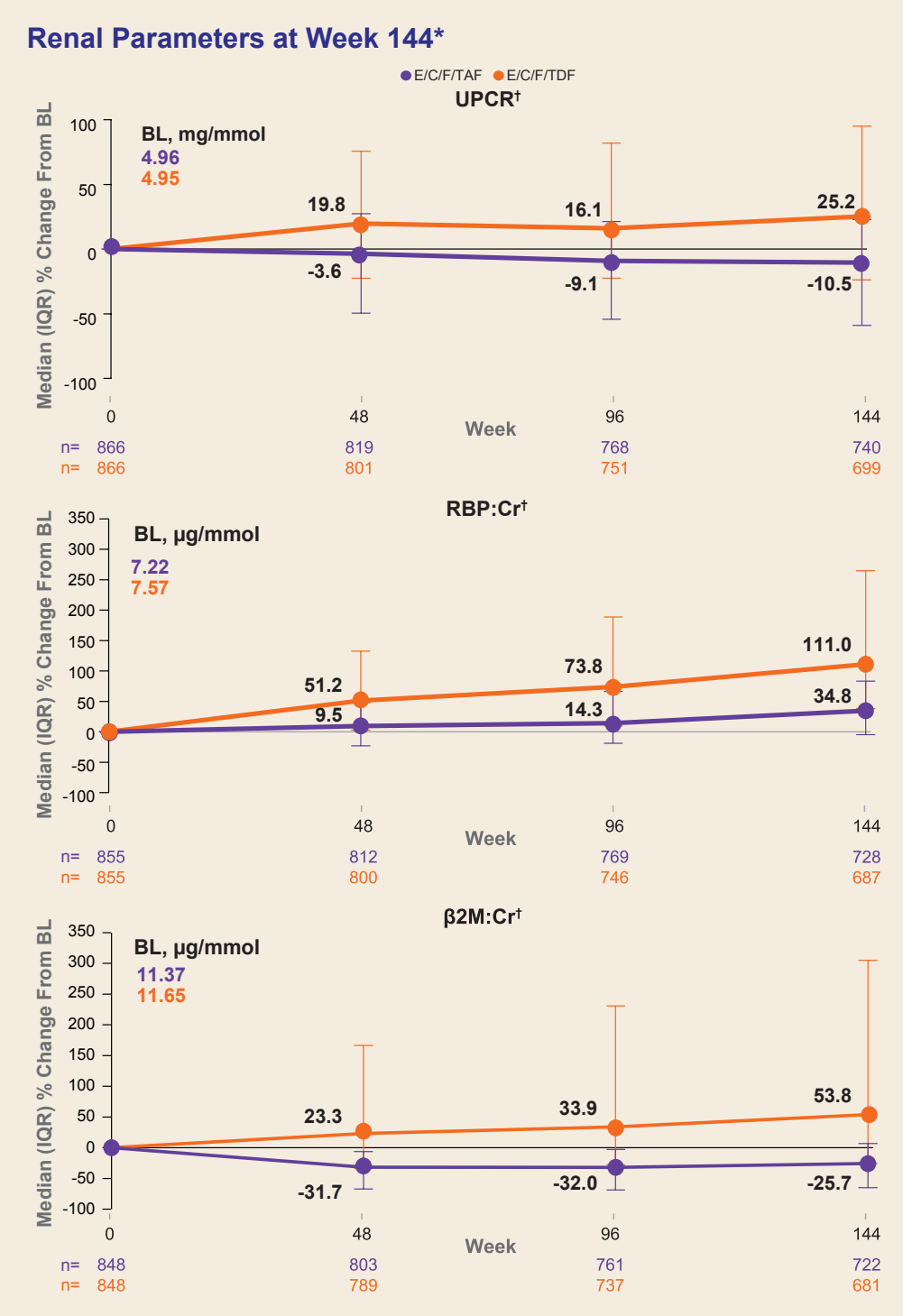

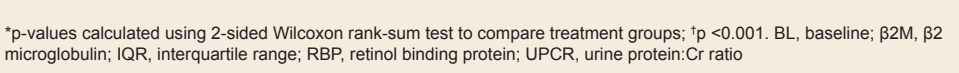
At Week 144, median change from baseline in eGFR $\mathrm{R}_{\mathrm{CG}}$ was significantly lower with E/C/F/TAF vs E/C/F/TDF (1.6 vs $7.7 \mathrm{~mL} / \mathrm{min}$ : significant
$\mathrm{p}<0.001)$

Week 144 Grade 3 or 4 Laboratory Abnormalities

\begin{tabular}{|c|c|c|}
\hline Partiipants, $\mathbf{n}(\%)$ & $\begin{array}{c}E / C / F / A F \\
n=862^{*}\end{array}$ & $\begin{array}{c}\mathrm{E} / \mathrm{C} / \mathrm{F} / \mathrm{TDF} \\
\mathrm{n}=865^{*}\end{array}$ \\
\hline Any Grade 3 or 4 lab abnormalities & $284(32.9)$ & $266(30.7)$ \\
\hline Creatine kinase elevation & $99(11.5)$ & $87(10.1)$ \\
\hline LDL elevation (fasting) & 92/839(11.0) & $40 / 834(4.8)$ \\
\hline Lipase $^{ \pm}$ & $6 / 127(5)$ & $13 / 154(8)$ \\
\hline Hypercholesterolemia (fasting) & $34 / 839(4.7)$ & $23 / 835(2.8)$ \\
\hline AST & $29(3.4)$ & $32(4.0)$ \\
\hline Amylase & $22(2.6)$ & $43(5.0)$ \\
\hline Hematuria (quantitative) & $25(2.9)$ & $26(3.0)$ \\
\hline Neutropenia & $16(1.9)$ & $26(3.0)$ \\
\hline
\end{tabular}

Median Change in Spine and Hip BMD through Week 144 Spine

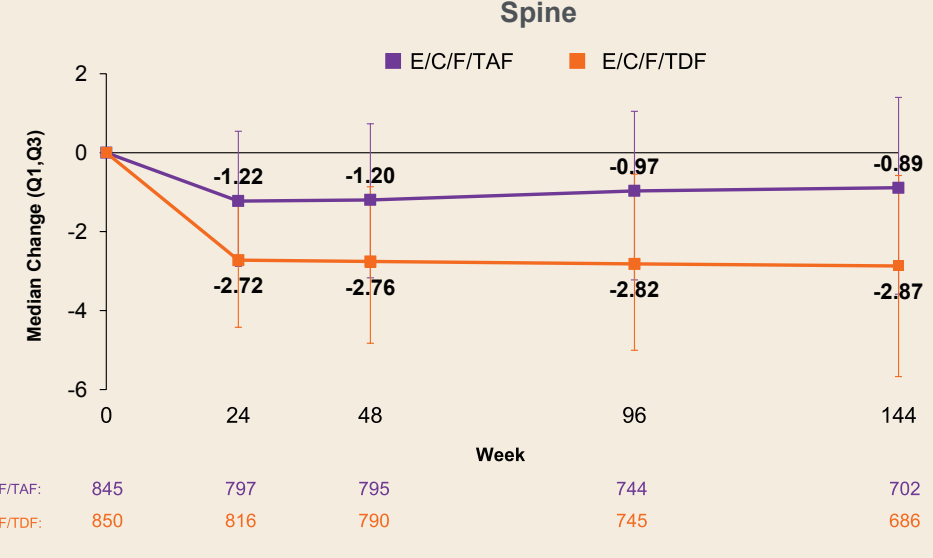

Hip

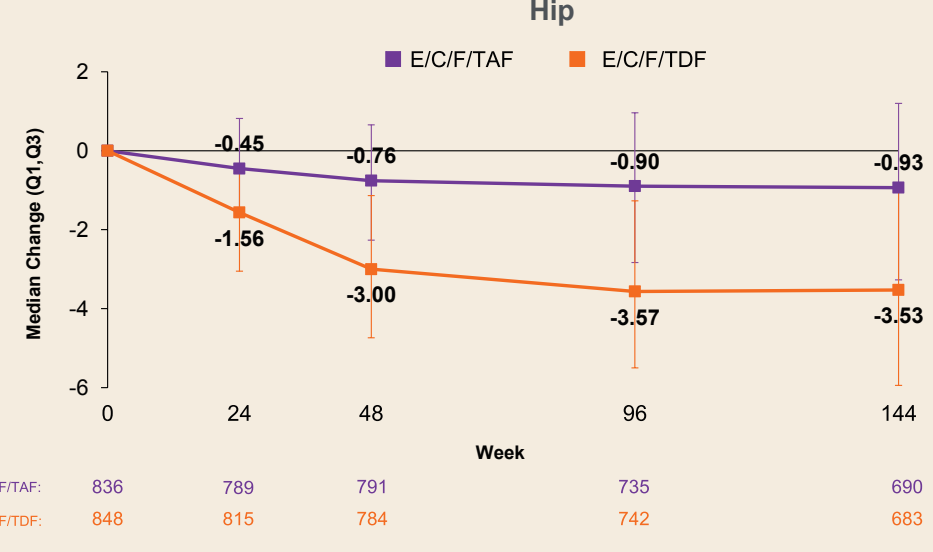

- \% initiating meds during study to increase BMD: TAF $16 \%$ vs. TDF $21 \% ; p=0.018$

Fasting Lipids Through Week $144^{*}$
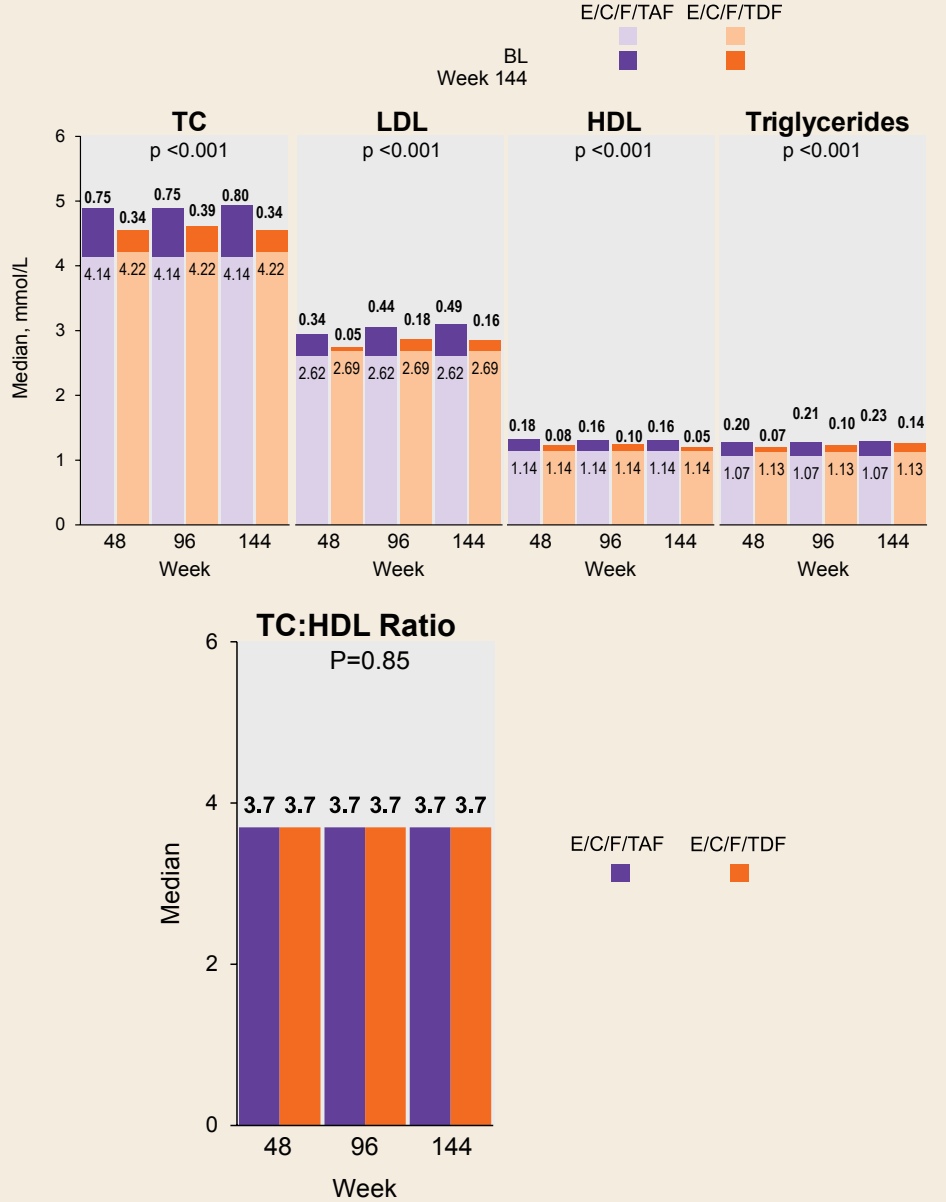

Participants on E/C/F/TAF had greater increases in TC, LDL, and HDL Pancipid-

\section{Conclusions}

- At Week 144, E/C/F/TAF was superior to E/C/F/TDF in virologic efficacy

- HIV RNA $<50$ copies/mL: $84 \%$ vs $80 \%$

- HIV RNA <20 copies/mL: $81 \%$ vs $76 \%$

- Emergence of resistance was rare

- E/C/F/TAF had significantly less impact than E/C/F/TDF on renal biomarkers

-0 vs 12 renal AEs; 0 vs 4 cases of proximal tubulopathy

- E/C/F/TAF had significantly less impact than E/C/F/TDF on BMD -0 vs $6 \mathrm{D} / \mathrm{C}$ for bone loss

- These longer-term data support the use of E/C/F/TAF as a safe well-tolerated, and durable regimen for initial and ongoing HIV-1
treatment

\section{References}

1. Sax P, et al. Lancet 2015;385:2606-15.
2. Wohl D et al. J Acquir Immune Defic Syndr 2016;72:58-64.
3. Sax P, et al. J Acquir Immune Defic Syndr 2014;67:52-8.

\section{Acknowledgments}

We extend our thanks to the participants, their families, and all participating 